\title{
SARS-CoV-2-associated Guillain-Barre syndrome is not infrequent
}

\author{
Josef Finsterer ${ }^{* *}$ (D), Fulvio Alexandre Scorza ${ }^{2}$ (i), \\ Carla Alexandra Scorza² (10, Ana Claudia Fiorini $i^{3,4}$ (1)
}

Dear Editor,

We read with interest the review article by Medeiros et al. ${ }^{1}$ about patients with SARS-CoV-2-associated Guillain-Barre syndrome (SAG) collected via a search of various databases. It was found that the main manifestations of SAG were fever, coughing, dyspnea, sore throat, ageusia, anosmia, and respiratory failure, in addition to paresthesias of the upper and lower limbs, quadruparesis, facial diplegia, areflexia, asthenia, mastoid pain, acute ataxia, fatigue, numbness, dysphagia, and moderate low back pain ${ }^{1}$. It was concluded that SARS-CoV-2 can trigger $\mathrm{GBS}^{1}$. This study is appealing but raises concerns that require discussion.

We do not agree with the notion that only few studies on the topic SAG are available as mentioned in the abstract and the discussion ${ }^{1}$. In a recent review about SAG, collecting available data until the end of July 2021, 120 articles reporting 300 patients with SAG were retrieved (Finsterer, submitted). The age of these patients ranged between 7 and 94 years. There was male preponderance. Latency between the onset of COVID-19 and SAG ranged between -10 and 90 days. Acute inflammatory demyelinating polyneuropathy (AIDP) was diagnosed in 171 patients, acute, motor axonal neuropathy (AMAN) in 24, and acute, motor, and sensory axonal neuropathy (AMSAN) in 16 patients. Intravenous immunoglobulins (IVIGs) were given to 241 patients, plasmapheresis to 28 patients, and steroids to 7 patients. A total of 59 patients were in need of artificial ventilation. Complete recovery was achieved only in 42 cases and partial recovery in 163 cases. Notably, 17 patients died.
The classification of GBS subtypes has been missed out in the review. GBS manifests with a variety of clinical presentations, such as AIDP, AMAN, AMSAN, Miller Fisher syndrome (MFS), polyneuritis cranialis (PNC), the pharyngo-cervico-brachial (PCB) subtype, and Bickerstaff encephalitis $(\mathrm{BFE})^{2}$. There are also patients with SAG who present with pure dysautonomia ${ }^{3}$.

The results of cerebrospinal fluid (CSF) findings have also been missed out. GBS may be characterized not only by the "dissociation cyto-albuminque" but also by the absence of virus mRNA in the CSF and elevated cytokines, particularly IL6, IL8, TNF- $\alpha$, and IL1 $b^{4}$ Only few patients have been reported in whom the CSF was positive for SARS-CoV-2 ${ }^{4,5}$, suggesting that SAG is truly immunogenic.

A discussion about GBS triggered by SARS-CoV-2 vaccinations is missing in the review. GBS may occur not only during COVID-19 infections but also after SARS-CoV-2 vaccinations. In a recent review about 19 gender-balanced patients with SARSCoV-2 vaccination-associated GBS (SVAG), aged 20-86 years, all patients developed SVAG after the first dose of the vaccine. The AstraZeneca vaccine was used in 14 cases, the Pfizer vaccine in 4 cases, and Johnson \& Johnson vaccine in 1 case. Latency between vaccination and the onset of SVAG ranged between $3 \mathrm{~h}$ and 39 days. SVAG patients responded favorably to IVIGs $(n=13)$ or steroids $(n=3)$. Six patients were in need of mechanical ventilation. Only one patient achieved complete recovery. Partial recovery was achieved in nine patients (Finsterer, submitted).

There is a discrepancy between the search dates given under "Information sources" (until April 2020) and the search date

\footnotetext{
${ }^{1}$ Klinik Landstrasse Hospital, Messerli Institute, Vienna - Vienna, Austria.

universidade Federal de São Paulo, Escola Paulista de Medicina, Disciplina de Neurociência - São Paulo (SP), Brazil.

${ }^{3}$ Pontifícia Universidade Católica de São Paulo, Programa de Pós-Graduação em Fonoaudiologia - São Paulo (SP), Brazil.

${ }^{4}$ Universidade Federal de São Paulo, Escola Paulista de Medicina, Departamento de Fonoaudiologia - São Paulo (SP), Brazil.

*Corresponding author: fifigs1@yahoo.de

Conflicts of interest: the authors declare there is no conflicts of interest. Funding: none.

Received on August 23, 2021. Accepted on September 07, 2021.
} 
given under "Search" (until June 26) without mentioning the year ${ }^{1}$. This ambiguity should be removed.

Overall, the review has several limitations that challenge the results and their interpretations. These limitations should be met to further strengthen the conclusions.

\section{AUTHORS" CONTRIBUTIONS}

JF: Conceptualization, Data curation, Validation, Writing - original draft. FS: Formal analysis, Validation, Writing - review \& editing. CS: Formal analysis, Validation, Writing - review \& editing. AF: Formal analysis, Validation, Writing - review \& editing.

\section{REFERENCES}

1. Medeiros KS, Macêdo LTA, Souza WF, Sarmento AC, Costa APF, Gonçalves AK. Guillain-Barré syndrome associated with SARS-CoV-2 infection: a scoping review. Rev Assoc Med Bras (1992). 2021;67(2):318-34. https://doi.org/10.1590/18069282.67.2.20200716

2. Finsterer J, Scorza FA. Guillain-Barre syndrome in 220 patients with COVID-19. Egypt J Neurol Psychiatr Neurosurg. 2021;57(1):55. https://doi.org/10.1186/s41983-02100310-7

3. Biassoni E, Assini A, Gandoglia I, Benedetti L, Boni S, Pontali $E$, et al. The importance of thinking about Guillain-Barré syndrome during the COVID-19 pandemic: a case with pure dysautonomic presentation. J Neurovirol. 2021;27(4):662-5. https://doi.org/10.1007/s13365-021-00997-7

4. Gigli GL, Vogrig A, Nilo A, Fabris M, Biasotto A, Curcio F, et al. HLA and immunological features of SARS-CoV-2-induced Guillain-Barré syndrome. Neurol Sci. 2020;41(12):3391-4. https://doi.org/10.1007/s10072-020-04787-7

5. Singh R, Shiza ST, Saadat R, Dawe M, Rehman U. Association of Guillain-Barre syndrome with COVID-19: a case report and literature review. Cureus. 2021;13(3):e13828. https://doi. org/10.7759/cureus. 13828 